\title{
Retraction
}

\section{Retracted: Research on Badminton Player's Step Training Model Based on Big Data and IoT Networks}

\author{
Security and Communication Networks
}

Received 15 November 2022; Accepted 15 November 2022; Published 27 November 2022

Copyright ( 2022 Security and Communication Networks. This is an open access article distributed under the Creative Commons Attribution License, which permits unrestricted use, distribution, and reproduction in any medium, provided the original work is properly cited.

Security and Communication Networks has retracted the article titled "Research on Badminton Player's Step Training Model Based on Big Data and IoT Networks" [1] due to concerns that the peer review process has been compromised.

Following an investigation conducted by the Hindawi Research Integrity team [2], significant concerns were identified with the peer reviewers assigned to this article; the investigation has concluded that the peer review process was compromised. We therefore can no longer trust the peer review process, and the article is being retracted with the agreement of the editorial board.

\section{References}

[1] L. Hao, J. Zhi, W. Zhu, and L. Zhou, "Research on Badminton Player's Step Training Model Based on Big Data and IoT Networks," Security and Communication Networks, vol. 2022, Article ID 1972389, 9 pages, 2022.

[2] L. Ferguson, "Advancing Research Integrity Collaboratively and with Vigour,” 2022, https://www.hindawi.com/post/advancingresearch-integrity-collaboratively-and-vigour/. 


\title{
Research on Badminton Player's Step Training Model Based on Big Data and IoT Networks
}

\author{
Long Hao, ${ }^{1}$ Juncai Zhi, ${ }^{2}$ Wei Zhu $\mathbb{D}^{3},{ }^{3}$ and Limin Zhou ${ }^{4}{ }^{4}$ \\ ${ }^{1}$ Institute of Physical Education and Training, Harbin Institute of Physical Education, Harbin 150006, Heilongjiang, China \\ ${ }^{2}$ Department of Physical Education, Tangshan Normal University, Tangshan 063000, Hebei, China \\ ${ }^{3}$ Ministry of Public Infrastructure, Guangxi Construction Vocational and Technical College, Nanning 530007, Guangxi, China \\ ${ }^{4}$ Institute of Physical Education and Training, Harbin Sport University, Harbin 150001, Heilongiang, China
}

Correspondence should be addressed to Wei Zhu; 2019210520@mail.chzu.edu.cn

Received 15 January 2022; Revised 27 January 2022; Accepted 5 February 2022; Published 26 February 2022

Academic Editor: Muhammad Arif

Copyright $(\odot 2022$ Long Hao et al. This is an open access article distributed under the Creative Commons Attribution License, which permits unrestricted use, distribution, and reproduction in any medium, provided the original work is properly cited.

In view of the poor effect of badminton players' physical fitness and response training, this paper puts forward the construction method of badminton players' pace training model based on big data, constructs badminton players' pace training index by combining big data technology, optimizes the pace training evaluation algorithm, and puts forward the corresponding Badminton Players' pace training method to achieve the model design goal. Finally, experiments show that the badminton athlete's pace training model based on big data has high practicability in the process of practical application and fully meets the research requirements.

\section{Introduction}

In addition to improving physical quality and response capacity, badminton has been shown to benefit both physical and mental wellness. More and more individuals are becoming interested in physical activity and participating in their favourite sports since the introduction of the notion of national sports was proposed. Through badminton practice, college students may alleviate the stress of academics while also balancing work and leisure time. The requirements for badminton teaching in colleges and universities are becoming more stringent as the educational reform process accelerates. Schools give close attention to the teaching of badminton to students [1]. They may improve the coordination of students' sports by increasing the intensity of footwork training, which will allow them to consistently increase the level of competition in badminton. It is necessary to develop footwork training in college badminton teaching in order to improve students' sensitivity and professional abilities, which is of significant practical value for college physical education. A major component of enhancing footwork training in college badminton instruction is to increase the overall teaching quality of badminton instruction [2]. Students' badminton technology may be considerably improved by increasing the intensity of their footwork training sessions. In badminton, only the combination of footwork and technique has the potential to significantly boost the sport's technological advancement. Badminton Footwork Training is the most important component in badminton technology. If kids do not get proper footwork instruction, it will have a negative influence on their overall badminton technical ability. Second, strengthening footwork training is also a vital requirement for ensuring the safety of players throughout the course of a sporting competition. The sport of badminton places tremendous demands on the physical fitness of its participants. Athletes should be able to maintain strong physical coordination abilities when participating in sports [3]. As a result, college physical education instructors should aggressively include footwork training into the badminton teaching process in order to raise the overall level of students' badminton performance. 


\section{Badminton Athlete's Step Training Model}

2.1. Badminton Athlete's Step Training Management Model. Badminton teaching footwork training refers to the special skill training mode that guides badminton trainers to improve their sports skills at the most scientific pace in badminton teaching. To be more specific, in badminton, badminton trainers may get the greatest result in badminton in the smallest amount of time, at the quickest pace, and with the most scientific approach via step training [4]. They may match the opponent's serve by transferring their weight effectively in order to gain the greatest sporting result. We can only provide high-quality training and achieve the required results if the training is of high quality. As part of pace training, each teacher should provide some high-quality recommendations to each kid in order for him or her to pay attention to nutrition and exercise in everyday life, maintain excellent physical health, and carry out pace instruction more effectively [5]. The training for badminton is distinct from other types of education. Students should be genuinely involved in it and actively connect with others. It is difficult to attain excellent achievements in any sport without putting in the necessary time and effort. Therefore, virtual reality technology can only be used as an auxiliary training of traditional badminton training methods, but can not replace traditional training [6]. Virtual reality technology also has its inherent advantages, which is of great positive significance to alleviate the tense field resources and coach resources. The implementation block diagram of virtual reality assisted badminton training is shown in Figure 1.

The big data algorithm is the basis of the subsequent recognition model. The quality of the preprocessing algorithm directly affects the recognition rate of the subsequent model. The original continuous data collected by the acceleration sensor $D$ is lengthy, including many actions and nonhitting data, noise, unwanted high-frequency information in the action gap, which is not conducive to the recognition of a single action. In addition, the original data is continuous, dense, and redundant [7]. It increases the time complexity of badminton stroke recognition algorithm and needs simplification, refinement, and dimensionality reduction [8]. As shown in Figure 2, data preprocessing mainly includes five steps: signal denoising, hitting action extraction, hitting action framing, eigenvalue selection, and vector quantization.

Following data gathering, the original data is denoised with the help of the signal denoising algorithm; after that, using window segmentation technology, the data information from a single stroke is recovered. A single striking action is also analysed in terms of its time sequence and frame, and many meta actions are dissected. The $K$-means clustering accuracy of numerous waveform feature quantities (such as peaks, peaks-plus-averages, and so on) for each meta action is evaluated; the feature quantity with the most visible clustering characteristics is selected as the feature information for each meta action. This is followed by vector quantization of each meta action and allocation of each meta action to a codebook space and then obtaining the observation sequence of each striking action to be used as input for the succeeding HMM algorithm model [9]. The signal component after multilayer wavelet transform is shown in the formula, where $s$ is the original signal and $t$ is the noise signal after the nth wavelet transform $E$. It is the effective signal after n-layer wavelet transform.

$$
W T(a, \tau)=\frac{1}{E-\sqrt{a}}+P \gamma-\int_{-\infty}^{\infty} f-\left(\frac{t-\tau}{\varphi}\right)
$$

where $P$ represents the original signal, $\gamma$ represents the effective signal, and $x_{i, j}$ is the signal length. The best denoising result is when SNR is the largest and MSE is the smallest. Assuming that each hitting action is decomposed into $n$ meta actions, the amount of data of each meta action is $100 / n$. According to the empirical value taken in this paper, each hitting action is decomposed into 10 meta actions. It is also a fixed length waveform [10]. By evaluating the clustering characteristics of its waveform eigenvalues, two eigenvalues with high clustering characteristics are selected as evaluation parameters.

$$
R=\frac{1}{\sum_{j=1}^{k} x_{i, j}}-\frac{\sum_{i=1} \lambda \gamma-k x_{i, j}}{W T(a, \tau)}
$$

Here, a formula is used to evaluate the clustering characteristics of the feature quantity, where $P\left(O^{(m)} \mid \lambda\right)$ is the number of clusters; $P_{m}$ is the total number of hitting action categories; $\beta_{t}^{(m)}$ Represents the number of actions of class $t$ recognized as class $m$, and the formula $\alpha_{t}^{(m)}$ represents the average clustering characteristic value. The recognition process of badminton action is to extract the observation sequence of the current hitting action through the preprocessing algorithm as is the input of each established hitting action hidden Markov model and use the Viterbi algorithm to obtain the occurrence probability of the best state sequence of the current hitting action under each model [11]. The recognition result of the present observation sequence is the striking action that corresponds to the model with the highest likelihood of producing the desired output result. Given that many data sets for the same striking action are present in the sample and that the HMM parameter model is trained on a single sample, the model data may fall into local optimum 2 , and the recognition rate of additional samples may be low [12], as previously stated. Two approaches are offered in order to prepare for the first mock test. The first is known as the mean training approach. It is necessary to combine data in order to provide the model with its input, which is the sample's observation sequence. The frequency weighted training approach is utilised to linearly weight how often each sample observation sequence is seen to improve the model's predictability. The formula of Welch training algorithm is modified. Let $m$ observation sequences $y_{1}^{(m)}(j)$ and $x_{1}^{(m)}(i)$ be the frequency of the $m$-th observation sequence. The correction formula is as follows:

$$
\overline{a_{i j}}=\frac{x_{1}^{(m)}(i)-y_{1}^{(m)}(j)}{\sum_{m=1}^{M} P_{m}-\alpha_{t}^{(m)} \beta_{t}^{(m)}}-R \sum_{m=1}^{M} \frac{1}{P\left(O^{(m)} \mid \lambda\right)} .
$$

It can be seen from the data that the frequency weighted training algorithm can effectively improve the recognition rate of the model, and the samples are trained through the 


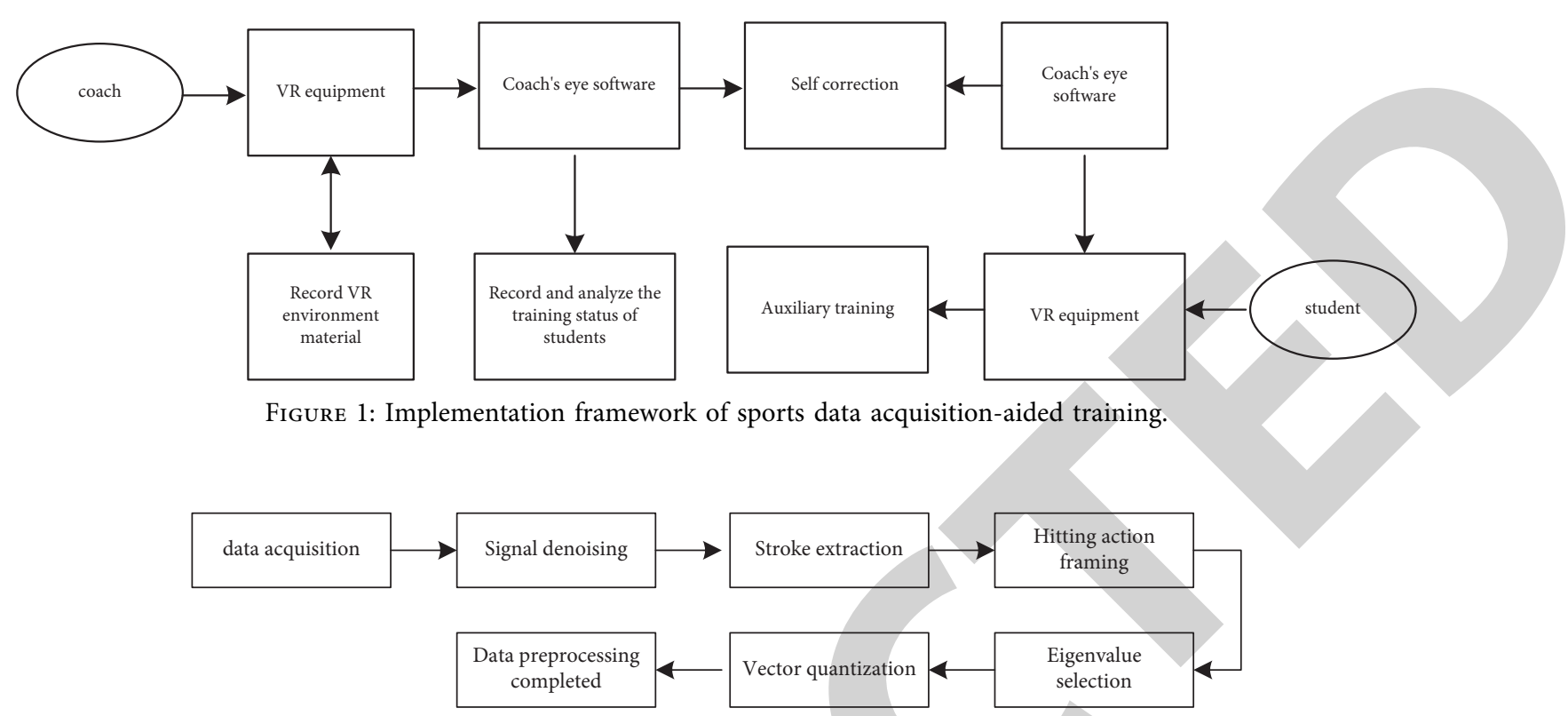

FIGURE 2: Data preprocessing process of badminton pace training.

training algorithm [13]. The training sample data is 100 shots of each of the ten serve actions. The corresponding model is obtained through the improved training algorithm. For any shot action to be identified, the observation sequence of the shot action is obtained through the big data algorithm. The conditional probability of the best state sequence of the observation sequence under the ten shot action models is calculated through the Viterbi algorithm [14]. Find the model corresponding to the maximum conditional probability, and the hitting action corresponding to this model is the recognition result. The hitting speed is estimated by the angular velocity data collected by the six-axis sensor fixed at the end of the racket. The peak value of the data of each shot is detected [15]. The peak time represents the shot time. Set the shot time as $t$, and then the instantaneous angular velocity at the shot time is obtained. As shown in the formula, $Q$ is the angular velocity at the time and the angular velocities on the $x, y$, and $z$ axes at the time. Taking the distance from the center of the racket surface to the tail end as $l$, the expression of the linear velocity $V$ at the time of swing is obtained, as shown in the formula, where $T$ is the corresponding hitting speed.

$$
\omega\left(t_{0}\right)=\overline{a_{i j}}-\sqrt{Q \omega_{x}^{2}\left(t_{0}\right)+V \omega_{y}^{2}\left(t_{0}\right)+T \omega_{z}^{2}\left(t_{0}\right)} .
$$

When mining association rules, we need to combine the data characteristics and adopt the data mining algorithm. The specific process is shown in Figure 3.

After querying and analyzing the data, it is necessary to collect the object related to the target and the reference set into the database. Association rules in database $X \Rightarrow Y$. The percentage of everything is called $X \Rightarrow Y$ expected confidence of. Comparing the confidence with the expected confidence, MBR technology is used to check the relationship between predicates, eliminate the predicate relationship that does not conform to the actual situation, and then form a topology data table, so as to calculate the support of predicates, exclude items with small support, and then form an optimal database. Generalize the topological relationship in the fourth step to form a new topological relationship data table, and then complete the data mining [16].

2.2. Evaluation Algorithm of Badminton Players' Pace Training. In the process of badminton teaching, teachers should gradually guide students to master the ways and methods to solve problems, and let students directly participate in exploration teaching and give full play to students' subjective initiative. Teachers' teaching method is the embodiment of each student's learning ability [17]. It is highly vital to students that their teachers have their own teaching methods. For every college student, what they pay attention to after class is practice rather than the continual infusion of academic information. On the other hand, some instructors use a strategy that involves the continual intake of academic information. They will not be able to boost students' learning abilities unless they include practical theory into their lessons. Instead, pupils would feel bored and pointless. Because of this, instructors should place a strong emphasis on practise and guidance [18]. The training procedure is scheduled to take eight weeks and include 32 hours of classroom instruction. The badminton courts and badminton rackets utilised for training in the experimental and control groups are identical, and the training period is also evenly scheduled in the morning to ensure that the experiment is as homogeneous as possible. It is my responsibility to monitor and oversee the whole procedure of the experiment, and each experimental element is rigorously controlled [19]. The test and statistics are carried out once the training is done. The statistical data are evaluated and concluded by vertical comparisons within the group and 


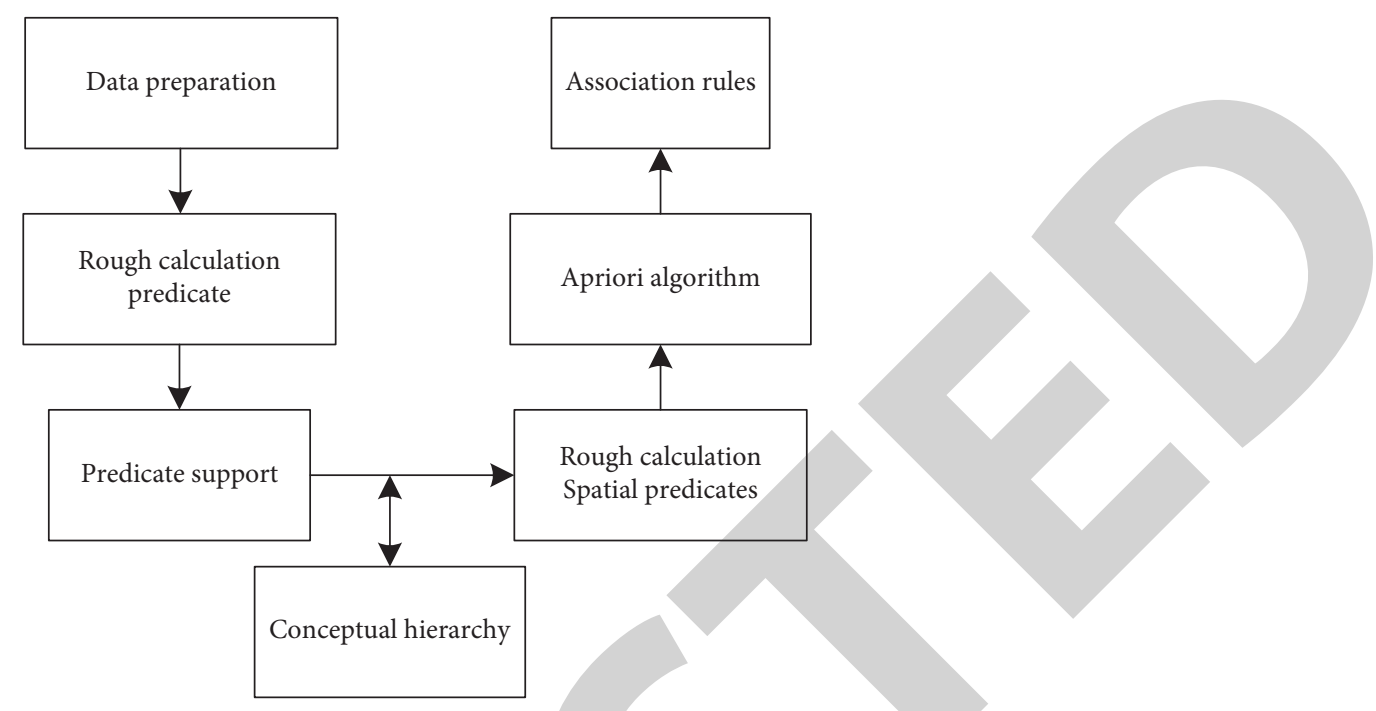

Figure 3: Specific process of badminton training data mining algorithm.

horizontal comparisons between the groups after the completion of the training. The overall experimental flow is shown in Figure 4.

In the traditional badminton singles teaching, single footwork training and combined footwork training are the key links, which need repetition and long-term practice. Okumura Hata intelligent model is the most widely used in the big data environment. In [20], the most important aspect of the model is that it has a high resistance to interference from external influences, which may assure the correctness of the system design. Okumura Hata's intelligent model is capable of formulating acquired data in a systematic manner and analyzing and interpreting the data properties in real time. Using the Okumura coordinate system diagram, the Okumura Hata model splits data into categories, and the link between characteristic data may be formed using the normal distribution of curve function. Facilitate intelligent progress data collection and establish Okumura Hata intelligent model. Firstly, analyze the intelligent extreme value of the collected data. The process is as follows:

$$
\theta=s_{B}\left[\frac{\mathrm{Q}(\mathrm{k})}{N(\mathrm{k}) M(\mathrm{k})}\right]-\omega\left(t_{0}\right)\left[\frac{K(\mathrm{k})}{\left[\omega_{x}^{2}\left(t_{0}\right)-\omega_{y}^{2}\left(t_{0}\right)-\omega_{z}^{2}\left(t_{0}\right)-1 \mid\right.}\right],
$$

where $Q(\mathrm{k})$ represents the range of modeling data; $N(\mathrm{k})$ indicates the foundation height; $M(\mathrm{k})$ indicates the operating frequency; $K(\mathrm{k})$ represents the batch corrected data model dynamics of loss index; $s_{B}$ represents the quantitative constant. The design of specific feedback data can be expressed as

$$
H[\mathrm{a}]=\omega_{x}^{2}\left(t_{0}\right)-\omega_{y}^{2}\left(t_{0}\right)-\omega_{z}^{2}\left(t_{0}\right)-\sum_{i=1}^{n} \mathrm{e}_{\mathrm{k}} \mathrm{w} \sigma^{\xi 2 i / 2},
$$

where $\sigma$ is the lag parameters representing the characteristics of athletes during training; $w$ represents the label operator. $e_{\mathrm{k}}$ is the main process to realize the intelligent formulation of training schedule. After the above steps, we can completely win the preliminary establishment of
Okumura Hata model. In the big data environment, there will be data transition in the process of athlete training data collection and intelligent data feedback. In order to prevent data transition, the calculation process of athlete training process needs to be corrected. The correction methods are as follows:

$$
\frac{\partial^{2} N_{2}}{\partial i^{2}}=\frac{H[a]}{Q(k)}-s_{B}\left[\frac{1}{N(k)+M(k)}\right] .
$$

In the correction process, the robustness between modules will be reduced, resulting in the increase of operation time. In order to solve this problem, the calculation process needs to be automatically adjusted and optimized. The total amount displayed in the proposed process of bipolar limit data is equivalent to the limit value. The maximum and minimum values of data need to be adjusted and optimized before they can be confirmed and expressed by the following formula:

$$
G_{f}=\frac{\partial^{2} N_{2}}{\partial i^{2}}-E_{P} \times \frac{1}{P\left(O^{(m)} \mid \lambda\right)},
$$

where $E_{P}$ represents the maximum critical value of the basic proposed total amount. The calculation correction and optimization in the intelligent customization of training progress are accomplished by using the aforementioned formula, which assures the efficacy and applicability of individualised training modification while also reducing costs. The long-term fundamental training is always tedious. If more interesting training methods can be developed in footwork teaching to ensure that the training is no longer boring, the training enthusiasm of students can be stimulated to the greatest extent possible; if a comprehensive training plan can be developed that can coordinate the overall rhythm of athletes and improve their physical quality, the training effect of footwork will be comprehensively strengthened. As a result, since the present footwork training techniques are not aimed at the sport of badminton 


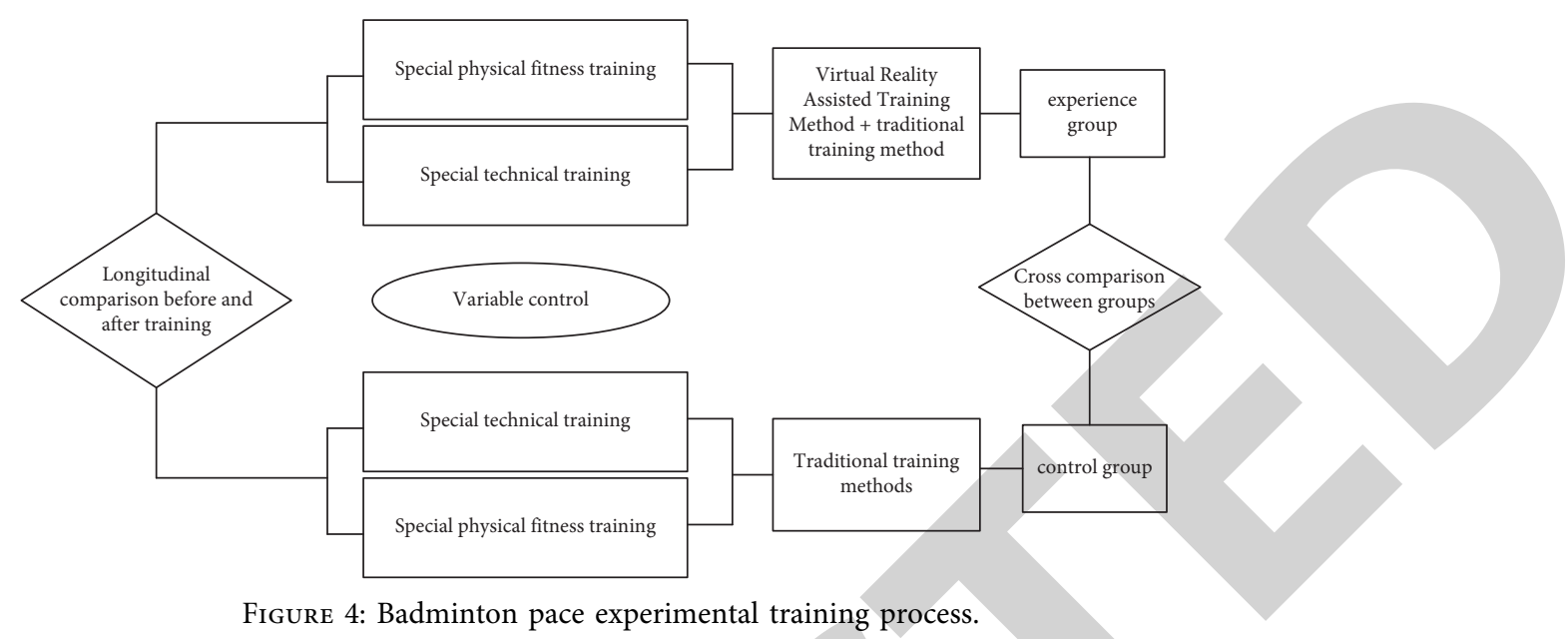

and do not provide more meaningful training, we often witness adult trainers exercising their footwork on the badminton court while playing the full field singles. In situations such as ball training or conflict, they are putting in twofold effort. Athletes' abilities may be developed in a variety of ways via varied focus training approaches, which can lead to more optimal training outcomes. The experimental scheme developed by the author for this investigation is shown in Table 1.

At the beginning of training, we should first guide students to understand badminton and master the relevant background knowledge, competition rules, referee methods, and sports spirit of badminton. In view of the characteristics of poor concentration and easy distraction of teenagers, the course teaching at this stage should not be too boring. Some video animation and badminton games can be integrated into the training process. Nowadays, smart phones have been popularized. Coaches can edit some badminton-related videos for students to watch to teach students relevant contents more clearly and guide them to understand and master the theoretical knowledge of badminton. The explanation of venues, rules, and other details should be combined with the actual situation, not on paper. Some small badminton games can also be used to deepen the students' impression and better guide the students' initiative. Badminton is a self height antagonistic sport. It is easy to get injured or accidently hurt teammates in training. Coaches should teach students basic injury handling skills and strictly declare classroom discipline. At this stage, coaches should also teach the basic grip method of badminton. The correct grip is the starting point of badminton. Coaches should observe each student patiently to ensure that each student has a good start. See Table 2 for the specific implementation scheme.

Badminton physical training should pay more attention to methods and processes. Physical training is a relatively boring and arduous process, but it can also help young athletes cultivate their brave and tenacious perseverance. It is also the formation process of young badminton players' competition style, which can lay a solid foundation for technical training and maintaining a good attitude in badminton. Physical quality training is a step-by-step process. It is forbidden to be eager for success and encourage young people. For juvenile training, special physical training should be gradually added on the basis of general physical training, supplemented by some interesting guidance to stimulate students' interest 48490 . See Table 3 for physical fitness training program.

They may carry out their instruction in real-world circumstances and lead and build independent thinking abilities in students while also stimulating their choice and excitement for physical activity. Furthermore, when carrying out teaching activities, physical education teachers can integrate theory with practise and contact their own closely related life cases, allowing students to integrate physical training into all aspects of daily life and apply what they have learned, resulting in a significant improvement in students' physical training skills over a short period of time.

\section{Analysis of Experimental Results}

Before the training, the physical quality of all students was assessed. The test method was 30 seconds' single swing rope skipping counting. Rope skipping can comprehensively investigate the students' jumping ability, coordination, and explosive power. In view of the physical characteristics of 8-12 year-old teenagers, 30 seconds was selected as the counting interval. During the training, a tracking file shall be established for each student, and the learning situation of each student shall be recorded for analysis and evaluation. It is separated into many categories, including physical fitness testing, technical action assessment, and a high and far ball fixed-point hitting exam, as well as a high and far ball movement hitting test, after the training session. The physical fitness test is divided into several categories, including 30-second single swing rope skipping, standing long jump, badminton long throw, and the whole field meter character step: the technical action test is evaluated by the coaches in detail, including the students' grip posture, hitting action, and step coordination; the effective hitting quantity of the trainees shall be used as the evaluation 
TABLE 1: Targeted training plan of badminton singles footwork.

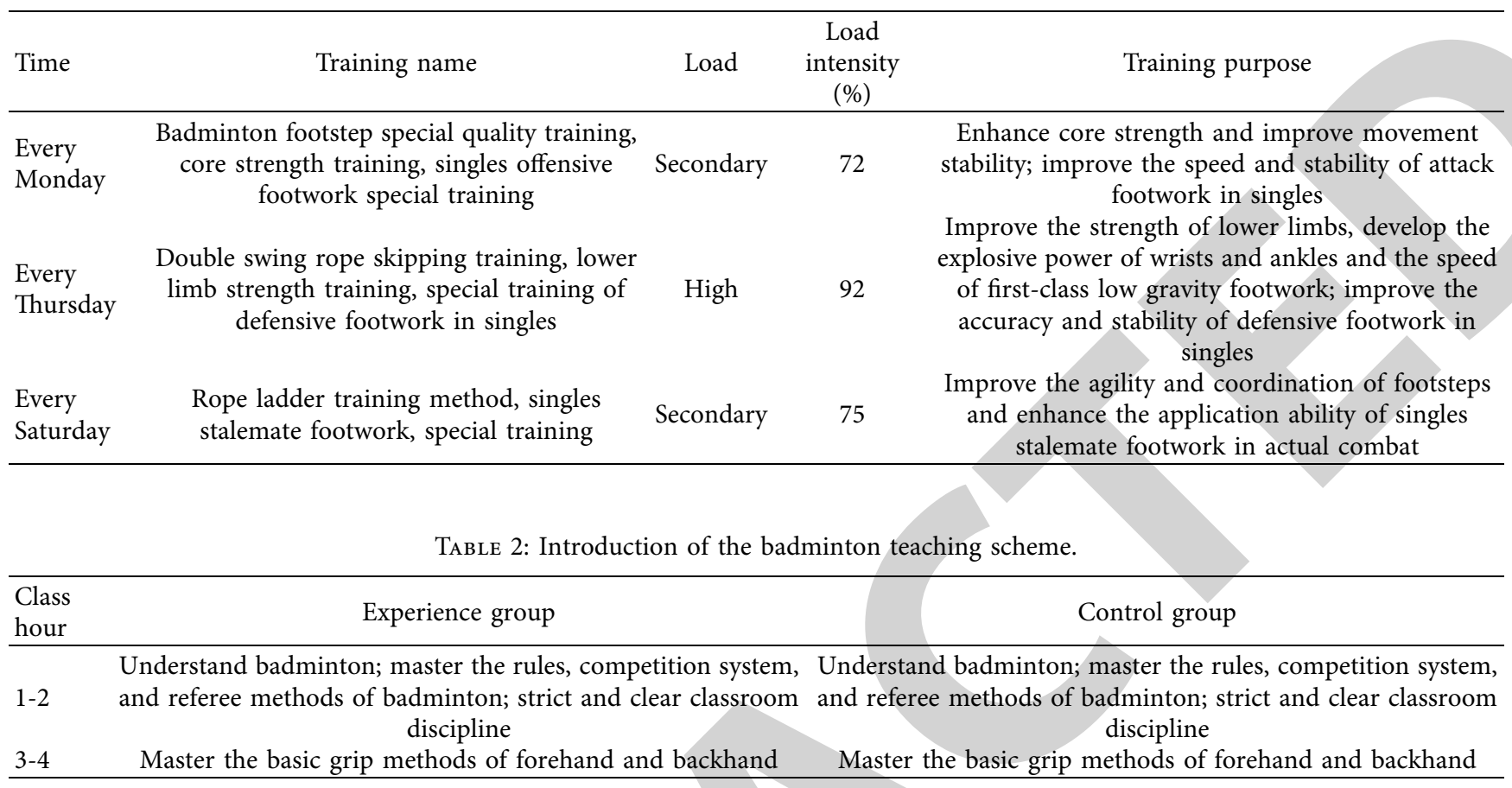

TABLE 3: Physical fitness training program of children's badminton.

\begin{tabular}{|c|c|c|}
\hline $\begin{array}{l}\text { Class } \\
\text { hour }\end{array}$ & Experience group & Control group \\
\hline $5-6$ & $\begin{array}{l}400 \mathrm{~m} \text { jogging in the field; measure the tension of leg ligament; } \\
\text { chest expansion shoulder joint training }\end{array}$ & $\begin{array}{l}400 \mathrm{~m} \text { jogging in the field; measure the tension of leg ligament; } \\
\text { chest expansion shoulder joint training }\end{array}$ \\
\hline $7-8$ & $\begin{array}{c}400 \mathrm{~m} \text { jogging in the field; high leg lifting training; broken line } \\
\text { round trip }\end{array}$ & $\begin{array}{c}400 \mathrm{~m} \text { jogging in the field; high leg lifting training; broken line } \\
\text { round trip }\end{array}$ \\
\hline $9-10$ & $\begin{array}{l}\text { VR badminton long throw training; single swing rope skipping } \\
\text { training; high leg lifting training; 600-meter jogging }\end{array}$ & $\begin{array}{l}\text { Badminton long throw training; single swing rope skipping } \\
\text { training; high leg lifting training; 600-meter jogging }\end{array}$ \\
\hline
\end{tabular}

standard for the fixed-point hitting of high-distance ball and the mop-up hitting of high-distance ball. The specific evaluation rules are shown in Tables 4 and 5. All assessment items and test standards are shown.

According to the results of a discussion with Badminton Coaches and the characteristics of teenagers, three items of standing long jump, $30 \mathrm{~s}$ single swing rope jump, and badminton long throw are selected as the physical fitness test content. The results of the test are compared using an independent sample $t$-test using spss20 to determine whether the experimental group and the control group are physically similar. See Table 6 for test result analysis.

Before the training, the badminton learning experience of the experimental subjects was understood through classroom inquiry, questionnaire survey and after-school interview with students' parents. The students with learning experience were further observed and screened to ensure that their existing learning experience would not interfere with the experimental results. The survey results are shown in Table 7.

The "just jump" bounce test is adopted. It adopts voltage conduction and is equipped with receptors, which can combine the felt stimulation with the computer and export it. This paper uses the data of time to evaluate the explosive force of subjects' lower limbs, as shown in Table 8 .

During training, the muscle fibers in the muscle become thicker, which leads to the increase of the cross-sectional area of the muscle, promotes the increase of the contraction strength of the muscle, and then improves the explosive power of the lower limb muscles and improves the subjects' knee flexion and vertical jump time. The knee flexion and vertical jump ability of the vibration training group ( $45 \mathrm{~Hz}$ and $30 \mathrm{~Hz}$ ) are higher than those of the traditional strength training group. When $45 \mathrm{~Hz}$ vibration training stimulation is compared to $30 \mathrm{~Hz}$ vibration training stimulation, it can be found that $45 \mathrm{~Hz}$ vibration training stimulation has a greater impact on knee flexion and vertical jump ability. It is necessary to construct a footwork movement test technique with six striking spots in a meter-shaped field in order to evaluate the effectiveness and worth of two distinct footwork training methods. The following are the two points of forehand and backhanded in front of the net (intersection between singles sideline and front service line), two points of catching and killing on both sides of singles (intersection between middle of 
TABLE 4: Evaluation criteria for physical fitness training of children's badminton.

Project

$30 \mathrm{~s}$ single swing rope skipping

Standing long jump

Badminton throw far

The pace of "meter" in the audience
Evaluation criterion

Direct counting

Direct counting

Direct counting Direct timing

TABLE 5: Evaluation criteria for counting movements of children's badminton.

\begin{tabular}{|c|c|}
\hline Score & Evaluation criterion \\
\hline 10 points & The posture is correct, standard, can accurately hit the badminton, and the hitting stability is good \\
\hline 9-8 points & Correct posture, consistent movement, accurate concentration of badminton, good stability \\
\hline 8-7 points & $\begin{array}{c}\text { The posture is correct, the basic movements are coherent and can not hit the badminton completely and accurately, and the } \\
\text { stability is general }\end{array}$ \\
\hline 6-5 points & Correct posture, incoherent basic movements, unable to hit the badminton completely and accurately \\
\hline 4-3 points & The posture is basically correct, the lead racket is not correct, and the badminton cannot be hit completely and accurately \\
\hline $\begin{array}{l}0-2 \\
\text { points }\end{array}$ & Incorrect posture, unable to accurately hit the badminton \\
\hline
\end{tabular}

TABLE 6: Independent sample $t$-test table of physical fitness test results of the experimental group and control group before the experiment $(m \pm s)$.

\begin{tabular}{lcccc}
\hline & $N$ & Standing long jump $(\mathrm{cm})$ & 30 s rope skipping (times) & Badminton throw distance (m) \\
\hline Experience group & 40 & $129.86 \pm 13.85$ & $45.65 \pm 7.51$ & $3.65 \pm 0.65$ \\
Control group & 40 & $128.32 \pm 18.58$ & $42.65 \pm 7.25$ & $3.62 \pm 0.52$ \\
$T$ & - & -1.625 & -1.656 & 0.658 \\
$P$ & - & 0.298 & 0.658 & 0.465 \\
\hline
\end{tabular}

TABLE 7: Statistics of badminton learning experience of experimental subjects.

\begin{tabular}{|c|c|c|c|c|c|c|}
\hline & \multirow[t]{2}{*}{$N$} & $\begin{array}{c}\text { More than } 3 \text { months of learning } \\
\text { experience }\end{array}$ & \multicolumn{2}{|c|}{ Study experience within 3 months } & \multicolumn{2}{|c|}{ Never touched } \\
\hline & & Number of people Proportion (\%) & Number of people & Proportion (\%) & Number of people & Proportion (\%) \\
\hline Experience group & 40 & 0 & 3 & 15.00 & 38 & 95.00 \\
\hline Control group & 40 & 50 & 3 & 7.50 & 37 & 92.50 \\
\hline Total & 80 & 2.50 & 6 & 7.50 & 75 & 93.75 \\
\hline
\end{tabular}

TABLE 8: Comparison of indexes among three groups of subjects before and after the experiment $(s)(n=21)$.

\begin{tabular}{lcccc}
\hline Experimental comparison & $T / P$ & Group A vs. group B & Group A vs. group C & Group B vs. group C \\
\hline \multirow{2}{*}{ Before the experiment } & $T$ & 0.752 & 1.852 & 2.185 \\
\multirow{2}{*}{ After the experiment } & $P$ & 0.185 & 0.865 & 0.265 \\
& $T$ & 1.625 & 4.152 & 3.058 \\
\hline
\end{tabular}

field and singles sideline), and two points of forehand and backhand in the back court (the intersection of singles sideline and doubles back service line) Each of the six points has three badminton balls positioned in front of it. The pupils who will be tested begin to make their way away from the middle of the field. The player must return to the center of the field utilising return footwork after placing a ball and then go on to the next ball. Move the badminton in a clockwise direction until the countdown of all 18 badmintons has come to an end. The findings of the meter step displacement timing test performed on the experimental group and the control group before and after the experiment are presented in Table 9 before and after the experiment.

After three months of different footwork training, the meter step displacement speed of the experimental group with targeted singles special footwork training and the control group with conventional footwork training were tested. The experimental results showed that there was a significant difference between the control group and the experimental group after training $(P<0.005)$. To sum up, the training methods used in the experimental group can significantly promote the improvement of badminton 
TABLE 9: Statistical unit of time for meter step displacement of the experimental group and the control group before and after the experiment (s).

\begin{tabular}{lcrl}
\hline Time & Group & Average & $P$ value \\
\hline $\begin{array}{l}\text { Before the } \\
\text { experiment }\end{array}$ & Experimental group & 25.65 & \\
& $(n=8)$ & $P<0.08$ \\
Cofter the & Experimental group & 19.98 & \\
experiment & $(n=8)$ & $P<0.08$ \\
& Control group $(n=8)$ & 21.65 & \\
\hline
\end{tabular}

TABLE 10: Statistics of teaching competition results of the experimental group and the control group after the experiment.

\begin{tabular}{lccc}
\hline Group & Wins & Failed sessions & Winning rate (\%) \\
\hline Experience group & 8 & 3 & 76 \\
Control group & 3 & 8 & 26 \\
\hline
\end{tabular}

singles level, shorten the moving time of single training, but do not weaken the training intensity. Therefore, compared with the traditional singles training methods, the training method in this paper is more effective than the traditional routine footwork training methods. In order to verify that the teaching method of targeted singles special footwork training is more significantly improved than the practical level of conventional general footwork training, this paper also organized a badminton singles competition with a single game 21 point system to verify the conclusion of this paper. The results are shown in Table 10 .

As shown in the table, after adopting the new special training of singles footwork, the winning rate of the experimental group using the special training method is 50 percentage points higher than that of the control group. Although there is no teaching competition test before the experiment, there is no training difference and physical quality difference between the two groups in the early stage, but the experiment can show that the new quasi-ideal training method has a significant improvement effect. The comparison of technical scores between the experimental group and the control group shows that the improvement speed of students is very obvious, and the improvement speed of the experimental group is very significant. Therefore, the practice results of this paper are effective. The targeted special training plan can better improve the singles level of athletes. By improving the footwork movement level, we can use hand technology more for confrontation. The movement speed of athletes is positively related to the hitting quality, which improves the students' comprehensive competitive ability.

\section{Conclusion}

To sum up, strengthening the training of Badminton footwork plays an important role in improving students' badminton technology. Physical education teachers in colleges and universities should fully realize the importance of footwork training and strengthen students' physical quality exercise in the actual teaching process to make it better meet the requirements of badminton. At the same time, physical education instructors should arrange students to make preparations prior to the official Badminton Footwork Training in order to prevent safety mishaps during the training. Teachers of high school physical education should provide targeted training for students based on the four steps of Badminton footwork to help students better master a variety of Badminton footwork and then improve students' badminton technical level, according to the four steps of Badminton footwork. Innovation is the hope of the country and the future of the nation. The innovation of badminton pace is the future prospect of national badminton. Efficient teaching method is the most key and important factor to improve students' learning pace, and it is also the premise of innovation. Only by implementing the correct way can the value of badminton be reflected.

\section{Data Availability}

The data used to support the findings of this study are included within the article.

\section{Conflicts of Interest}

The authors declare that they have no conflicts of interest.

\section{References}

[1] S. Liu, D. Liu, K. Muhammad, and W. Ding, "Effective template update mechanism in visual tracking with background clutter," Neurocomputing, vol. 458, pp. 615-625, 2021.

[2] S. Liu, S. Wang, X. Liu et al., "Human memory update strategy: a multi-layer template update mechanism for remote visual monitoring," IEEE Transactions on Multimedia, vol. 23, pp. 2188-2198, 2021.

[3] S. Liu, T. He, and J. Dai, "A survey of CRF algorithm based knowledge extraction of elementary mathematics in Chinese," Mobile Networks \& Applications, vol. 26, no. 5, pp. 1891-1903, 2021.

[4] L. Kong and Y. Ma, "Big data adaptive migration and fusion simulation based on fuzzy matrix," Computer simulation, vol. 37, no. 3, p. 4, 2020.

[5] B Sánchez, P Abián, F. Jimenez, and J. Abián-Vicén, "Structural and mechanical properties of the Achilles tendon in senior badminton players: operated vs. non-injured tendons," Clinical Biomechanics, vol. 85, no. 2, Article ID 105366 , 2021.

[6] T. Steels, B. Herbruggen, J. Fontaine, T. Pessemier, and E. Poorter, "Badminton activity recognition using accelerometer data," Sensors, vol. 20, no. 17, 4685 pages, 2020.

[7] Z. Cao, T. Liao, W. Song, and Z. Chen, "Detecting the shuttlecock for a badminton robot: a YOLO based approach," Expert Systems with Applications, vol. 164, no. 4, 113833 pages, 2020.

[8] W. Chen, T. Liao, Z. Li et al., "Using FTOC to track shuttlecock for the badminton robot," Neurocomputing, vol. 334, pp. 182-196, 2019.

[9] R. Jie, F. Guan, M. Pang, and S. Li, "Monitoring of human body running training with wireless sensor based wearable devices," Computer Communications, vol. 157, no. 4, pp. 343-350, 2020.

[10] J. Lempke, J. M. Hart, D. J. Hryvniak, J. S. Rodu, and J. Hertel, "Use of wearable sensors to identify biomechanical alterations 
in runners with exercise-related lower leg pain," Journal of Biomechanics, vol. 126, no. 5, 1106 pages, 2021.

[11] I. C. Stanica, F. Moldoveanu, G. P. Portelli, M. I. Dascalu, and M. G Ristea, "Flexible virtual reality system for neurorehabilitation and quality of life improvement," Sensors, vol. 20, no. 21, 6045 pages, 2020.

[12] S. Glowinski, T. Krzyzynski, A. Bryndal, and I. Maciejewski, "A kinematic model of a humanoid lower limb exoskeleton with hydraulic actuators," Sensors, vol. 20, no. 6116, 6116 pages, 2020.

[13] A. Hojjati Najafabadi, S. Amini, and F. Farahmand, "The effect of saddle-assistive device on improving the gait parameters of patients with the lower limbs weakness: a pilot study," Journal of Bionics Engineering, vol. 17, no. 6, pp. 1175-1185, 2020.

[14] H. Y. Huang, I. Farkhatdinov, A. Arami, M. Bouri, and E. Burdet, "Cable-driven robotic interface for lower limb neuromechanics identification," IEEE Transactions on Biomedical Engineering, vol. 991 page, 2020.

[15] A. Jd, B. Dp, and A. Ks, “Assessment of HEADS UP online training as an educational intervention for sports officials/ athletic trainers," Journal of Safety Research, vol. 74, no. 3, pp. 133-141, 2020.

[16] J. L. Herold and A. Sommer, "A mathematical model-based approach to optimize loading schemes of isometric resistance training sessions," Sports Engineering, vol. 24, no. 1, pp. 1-15, 2021.

[17] A. Singh, S. Garg, K. Kaur, S. Batra, N. Kumar, and K. K. Raymond, "Fuzzy-folded bloom filter-as-a-service for big data storage in the cloud," IEEE Transactions on Industrial Informatics, vol. 15, no. 4, pp. 2338-2348, 2019.

[18] J. S. Guerrero-Prado, W. Alfonso-Morales, E. Caicedo-Bravo, B. Zayas-Pérez, and A. Espinosa-Reza, "The power of big data and data analytics for AMI data: a case study," Sensors, vol. 20, no. 11, 3289 pages, 2020.

[19] S. P. Rao and M. Kusuma, "Application-aware big data deduplication in cloud environment," IEEE Transactions on Cloud Computing, vol. 7, no. 4, pp. 921-934, 2019.

[20] R. M. A. Latif, S. B. Belhaouari, S. Saeed, I. Laiqa-Binte, and M. Farhan, "Integration of google play content and frost prediction using cnn: scalable iot framework for big data," IEEE Access, vol. 8, no. 99, 1 page, 2020.

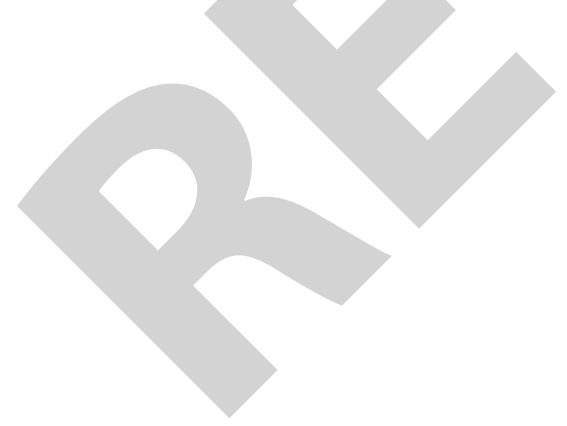

